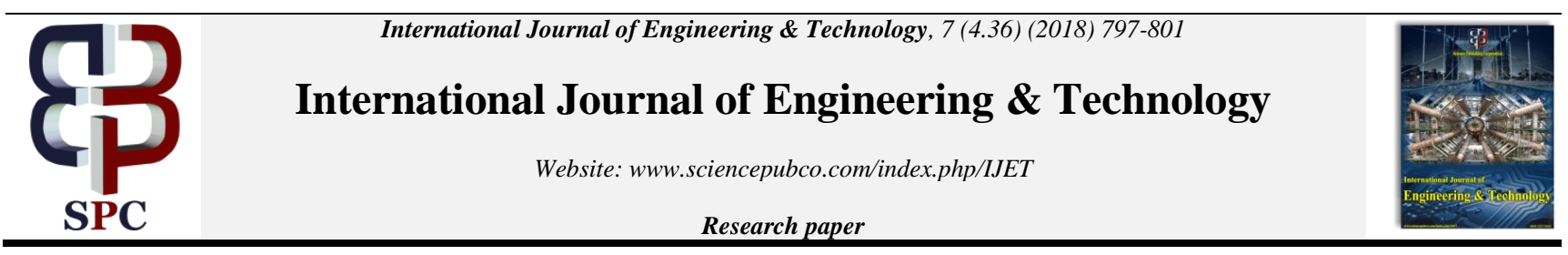

\title{
Multidimensional Data Analysis of Location Based Social Network
}

\author{
Shaik Mastan Vali ${ }^{*}$, P. Sujatha ${ }^{2}$ \\ ${ }^{1}$ Research Scholar, School of Computing Sciences, Vels Institute of Science, Technology \& Advanced Studies, Chennai. \\ ${ }^{2}$ Associate Professor, School of Computing Sciences, Vels Institute of Science, Technology \& Advanced Studies, Chennai. \\ E-mail: suja.research@gmail.com \\ *Corresponding author E-mail:Shaikmastan77@gmail.com
}

\begin{abstract}
Long range interpersonal communication benefits gather data on clients' social contacts, make an expansive interrelated informal organization, and open to clients how they are connected to others in the system. The basic of an OSN contains of customized client profiles, which for the most part encase interests (e.g. bought in intrigue gatherings), perceiving data (e.g. name and photograph), and individual contacts (e.g. rundown of connected clients, alleged "companions"). The ability to accumulate and inspect such information conveys particular chances to perceive the central belief systems of interpersonal organizations, their creation, movement and attributes. These sorts of informal communities are classified to be specific scholarly, general and area based interpersonal organizations. In this paper, we concentrated on the area based interpersonal organizations. Here, we investigations the diverse kinds of information that utilizations in area based interpersonal organizations and furthermore examine the effect of online datasets on neighborhood based interpersonal organization.
\end{abstract}

Keywords: Location based social networks, bright kite, Gowalla, comparison, impact of LBSN.

\section{Introduction}

An interpersonal organization is a social structure made up of people connected by means of at least one exact sorts of interdependency, similar to fellowship, common interests, and shared information [1]. More often than not, an informal communication benefit shapes on and mirrors the genuine interpersonal organizations among individuals through online stages, for example, a site, given that routes for clients to share thoughts, exercises, activities, and interests over the Web. The total openness of area securing innovation (for example GPS and $\mathrm{Wi}-\mathrm{Fi}$ ) approves individuals to improve an area measurement to existing on the web informal communities in a decent variety of ways.

In the useful world, various social, organic, and data frameworks can be normally characterized as perplexing systems in which hubs speak to substances (elements or organizations) and connections mean diverse relations between these elements [2]. An interpersonal organization is an unpredictable system in which hubs speak to people or different elements in a social setting, while joins speak to a relationship among them, similar to companionship, connection, collaboration or others. By expanding utilization of Web and cell phones, different web stages like Facebook, Twitter and Foursquare execute informal community environs focused at giving modified administrations to empower the connecting between people with comparative interests and exercises. These stages, likewise named as online interpersonal organizations (OSNs), have progressed toward becoming piece of the regular day to day existence of bunches of individuals around the globe who persistently keep and make new social connections. OSNs giving area based administrations to clients to registration a physical place are named area based informal organizations (LBSNs).

The progressions among various types of interpersonal organizations have been outlined, e.g., scholastic systems, for example, DBLP, general online informal organizations, as Facebook, and area based interpersonal organizations, for example, Foursquare and GeoLife, in Table 1. LBSNs current novel shots and difficulties given the restrictive properties of positions, the heterogeneous structure of a system, and their high rate of advancement and development.

Table 1: Comparison of Three Different Social Networks

\begin{tabular}{|l|l|l|l|}
\hline & $\begin{array}{l}\text { Location } \\
\text { Awareness }\end{array}$ & $\begin{array}{l}\text { Heterogeneous } \\
\text { Environments }\end{array}$ & $\begin{array}{l}\text { Evolving } \\
\text { speed }\end{array}$ \\
\hline $\begin{array}{l}\text { Academic Social } \\
\text { Networks }\end{array}$ & & Yes & Slow \\
\hline $\begin{array}{l}\text { General online } \\
\text { social networks }\end{array}$ & & & Fast \\
\hline $\begin{array}{l}\text { Location based } \\
\text { social networks }\end{array}$ & Yes & Yes & Fast \\
\hline
\end{tabular}

\section{Location-based social networks}

An area based informal community (LBSN), likewise specified to as geo-interpersonal organization or, geographic interpersonal organization and is authoritatively characterized as an exact kind of long range interpersonal communication stage in which geographic administrations supplement customary informal communities [3]. This additional data allows new social elements, and also those got from visits of clients to the comparable or same areas, in adding to data of general interests, exercises and practices 
gathered as of the arrangement of spots go to see by a man and the area labeled information delivered through these visits.

An area based interpersonal organization (LBSN), spoken to by an undirected system $\mathrm{G}(\mathrm{V}, \mathrm{E}, \mathrm{L})$, where $\mathrm{E}$ is the arrangement of edges instead of social connections among clients, $\mathrm{V}$ is the arrangement of clients, L is the arrangement of adjusted spots visited by each client, and is the arrangement of registration speaking to joins among clients and spots. This delineation duplicates the event of two sorts of hubs: clients and areas, and two sorts of connections: client area (registration) and client (social connections), or, in other words of the heterogeneity of LBSNs $[4,5,6]$.

The straightforward commence of latest LBSNs is very unassuming. Cell phone clients interrelate with and application so make their present area known on the system. Particulars of a client's area can be included one of two different ways: either physically - through a SMS instant message coordinated by the client or by means of the client plotting their situation on a guide or, as is getting more mainstream, the portable application can consequently close a client's current position by utilizing the intensity of a numeral area assurance information's (Furey et al., 2008; Furey et al., 2011). In latest LBSNs, the method of sharing our present area has turned out to be recognized as 'checking in'. Checking in is viewed as the fundamental client movement in adding to survey other clients' areas, thinking positions visited, et cetera. Contingent upon the administration, a client's shared position data can be seen by a subgroup of individuals buying in to the office or by somebody on the informal community who is resolved to be inside a specific region of the client.

In any case, as area data is a standard asset in LBSNs, modified creators have anticipated diverse techniques. The strategies used to foresee kinship joins, which are GPS and additionally registration history [7], land remove [8], area semantics (labels, classes, and so forth.) [9] and other versatility client designs as insights sources to advance the viability of fellowship expectation in LBSNs.

Google Scope is conceivably the humblest as far as its highlights. Its basic objective is to "Given you a chance to see where your companions are at this moment" [10].

To every client can ask for loved ones by a Google record to begin sharing their region. When clients are detectable to each other, each can see the other's area every last time they select to raise todate that information utilizing the Scope application introduced on their PC or cell phone.

Google propose a couple of humble approaches to make utilization of this capacity; keeping a past filled with our territories, getting alarms to our telephone that a companion is close or demonstrating our present territory as our status message on the Google Talk benefit, for example. Scope is an example of a LBSN which places significance on clients' control of their own area classification, allowing clients to just impart territories to pre approved connections and allowing area stowing away for distinct contacts.

\section{Analysis of datasets and data for LBSNs}

LBSN needs to be assessed which information is available in the online system and how we can access and utilize that information. Contingent upon the idea of the exact online system, the information classes will vary on sequent [11].

As indicated by our early on examinations, Foursquare and Facebook Places basically convey data on Purpose of Enthusiasm with the allowing properties, similar to address or opening hours, over data on the numeral visiting clients, while applications, for example, MapMy Tracks are focused on sportive occasions and will in this way convey straight information on climbing or biking courses with trouble levels or esteemed time length joined as property information.

\subsection{Nature of information}

Common area based person to person communication destinations allow clients to "check in" at a physical place and offer the region with their online companions, and solink the hole in the midst of the handy world and online informal organizations [12].

The accessibility of colossal measures of geological and social information on LBSNs conveys an extraordinary opportunity to contemplate human portable execution through information investigation in a spatial-transient social setting, allowing an assorted variety of area based administrations, from versatile advertising to catastrophe discharge.

A large portion of the dataset encases an entire data about the registration data, social data and place data. And furthermore the vast majority of the datasets are accessible on system archives.

\subsection{Examination of datasets}

The technique of evaluating information utilizing expository and coherent thinking to investigate each segment of the information conveyed. This type of investigation is one of the various advances that must be proficient while coordinating an exploration analyze [13].

Information from various sources is gathered, updated, and afterward concentrated to frame a type of disclosure or end. Likewise it is a methodology of efficiently applying measurable and additionally sensible strategies to characterize and show, consolidate and survey, and gauge information. It incorporates a few phases including building up an informational collection, making the information for handling, applying models, discovering key discoveries. The point of information examination is to find illicit dreams that can advise basic leadership.

\section{Dataset}

For dataset and information examination, we are pointing on the two sorts of LBSN datasets, in particular Brightkite and Gowalla, both the datasets are transparently accessible from the Stanford organize investigation venture (SNAP).

\subsection{Gowalla dataset}

Gowalla is an area based informal communication site where clients share their areas by checking-in. The fellowship organize is undirected and was as one utilizing their open Programming interface, and contains of 196,591 hubs and 950,327 edges. We have together an aggregate of $6,442,890$ registration of these clients over the time of Feb. 2009 - Oct. 2010.

This dataset was as one from Gowalla, a typical area based informal organization, which has in excess of 600,000 clients since November 2010 and was created by Facebook in December 2011. By and by, the Gowalla APIs used to accumulate the client profiles, client fellowship, area profiles, and clients' registration history made before June 1, 2011. Ultimately, got 36,001,959 registration made by 319,063 clients more than $2,844,076$ areas. The areas in Gowalla are amassed into 7 coregroups, i.e., Outside, Nourishment, Amusement, Nightlife, People group, Shopping and Travel, and each significant class contains of various subgroups.

\subsection{Bright kite dataset}

This undirected system contains user- client fellowship relations from Brightkite, a previous area based interpersonal organization were client shared their areas [15].

A hub means a client and an edge coordinates that a fellowship exists between the client indicated by the left hub and the client spoken to by the correct hub. 
The dataset contains of 4,491,143 open registration information accomplished by 58,228 Brightkite clients in 772,966 discrete spots, all through the period between April 2008 and October 2010. Brightkite clients additionally contribute in a fellowship arrange, which contains of 214,078 connections. It was begun in 2007 as a long range informal communication site which grants clients to impart their territory to their companions: it is offered worldwide and it is established on making registration at spots, where clients can see who is close and who has been there $\begin{array}{lllllllllll}\mathrm{p} & \mathrm{r} & \mathrm{e} & \mathrm{v} & \mathrm{i} & \mathrm{o} & \mathrm{u} & \mathrm{s} & \mathrm{l} & \mathrm{y}\end{array}$ Brightkite clients can make regular kinship connections and they can drive their registration to their Twitter and Facebook accounts. This dataset means an entire preview of a well known area based administration in its fundamental advancement stage.

\subsection{Detailed comparison of Gowalla and bright kite dataset}

Table 2: Comparison of Gowalla and brightkite datasets

\begin{tabular}{|c|c|c|}
\hline Parameters & Gowalla & Brightkite \\
\hline Vertex type & User & User \\
\hline Edge type & Friendship & Friendship \\
\hline Network type & Undirected & Undirected \\
\hline Edge weights & Un weighted & Unweighted \\
\hline No. of nodes & 196,591 users & 58,228 users \\
\hline No. of edges & $\begin{array}{l}950,327 \\
\text { friendship }\end{array}$ & $\begin{array}{l}214,078 \\
\text { friendship }\end{array}$ \\
\hline $\begin{array}{l}\text { Average no. of edges } \\
\text { attached to a vertex }\end{array}$ & $\begin{array}{l}9.66 \text { edges } \\
\text { vertex }\end{array}$ & $\begin{array}{l}7.35 \text { edges } / \\
\text { vertex }\end{array}$ \\
\hline $\begin{array}{l}\text { Ratio of edges to the total } \\
\text { no. of possible edges }\end{array}$ & $\begin{array}{l}4.917 \times 10^{2} \times \\
\text { edges / vertex }\end{array}$ & $\begin{array}{l}1.262 \times 10^{-4} \\
\text { edges / vertex }\end{array}$ \\
\hline $\begin{array}{l}\text { Highest no. of edges } \\
\text { attached to a node }\end{array}$ & 14,730 edges & 1,134 edges \\
\hline $\begin{array}{l}2 \text {-star consists of a node } \\
\text { connected to } 2 \text { other } \\
\text { nodes }\end{array}$ & $290,400,040$ & $13,423,403$ \\
\hline $\begin{array}{l}\text { A square is a cycle of } \\
\text { length four }\end{array}$ & $146,652,712$ & $23,380,701$ \\
\hline $\begin{array}{l}\text { Cyclical list of connected } \\
\text { vertices }\end{array}$ & $2,336,722,510$ & $241,167,376$ \\
\hline $\begin{array}{l}\text { Gini } \\
\text { coefficient }\end{array}$ & $68.3 \%$ & $68.5 \%$ \\
\hline $\begin{array}{l}\text { Clustering coefficient } \\
\text { (probability that } \\
\text { neighbors of a node are } \\
\text { connected) }\end{array}$ & $2.35 \%$ & $11.1 \%$ \\
\hline $\begin{array}{l}\text { Diameter of a } \\
\text { network }\end{array}$ & 16 edges & 18 edges \\
\hline Mean shortest pathlengh & 4.43 edges & 4.86 edges \\
\hline
\end{tabular}

\subsection{Graphical analysis of Gowalla and bright kite}

\subsubsection{Lorenz curve degree distribution}

The Lorenz bend is an instrument at first from financial aspects that imagines articulations of the shape "X\% of hubs with most reduced degree represent $\mathrm{Y} \%$ of edges". The arrangement of qualities $(\mathrm{X}, \mathrm{Y})(\mathrm{X}, \mathrm{Y})$ therefore characterized is the Lorenz bend. In a system the Lorenz bend is a straight askew line when all hubs have the comparable degree, and bended else. The territory in the midst of the Lorenz bend and the inclining is a large portion of the Gini coefficient.

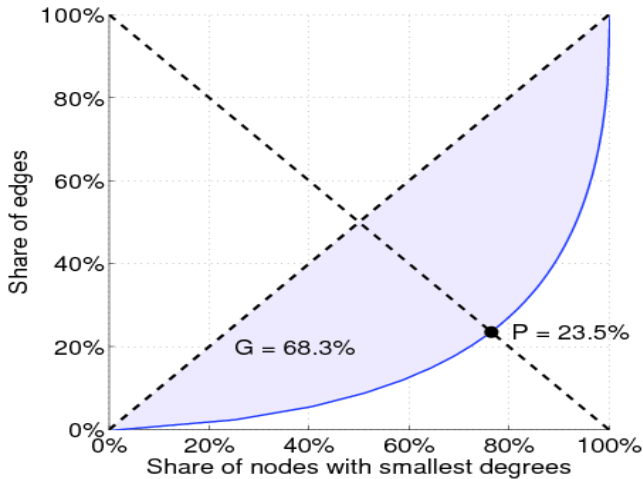

Figure 1: Lorenz curve degree distribution of Gowalla

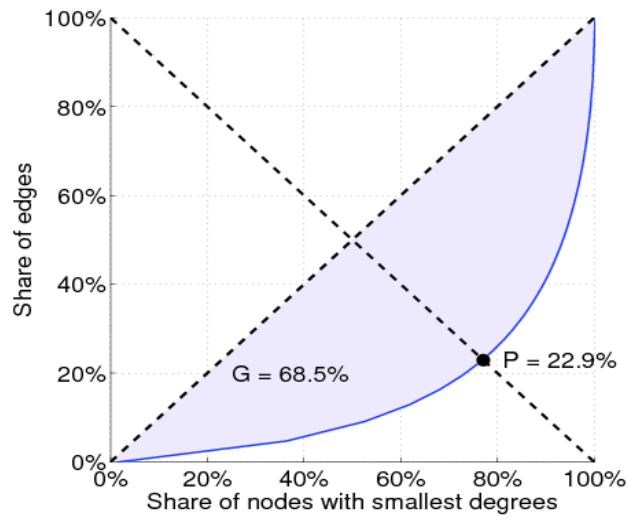

Figure 2: Lorenz curve degree distribution of Brightkite

\subsection{Distance distribution}

Separation measurements can be imagined out there circulation plot [16]. The separation conveyance plot shows, for each whole number $\mathrm{k}$, the quantity of hub sets at separation $\mathrm{k}$ from one another, partitioned by the aggregate number of hub sets. The separation appropriation plot can be utilized to peruse off the width, the middle way length, and the 90-percentile viable width. For ordered systems, the separation conveyance plot can be exhibited after some time.

The non-sequential separation conveyance plot shows the cumulated separation circulation work among all hub sets $(\mathrm{u}, \mathrm{v})$ in the system, with sets of the shape $(u, u)$, whose separation is zero.

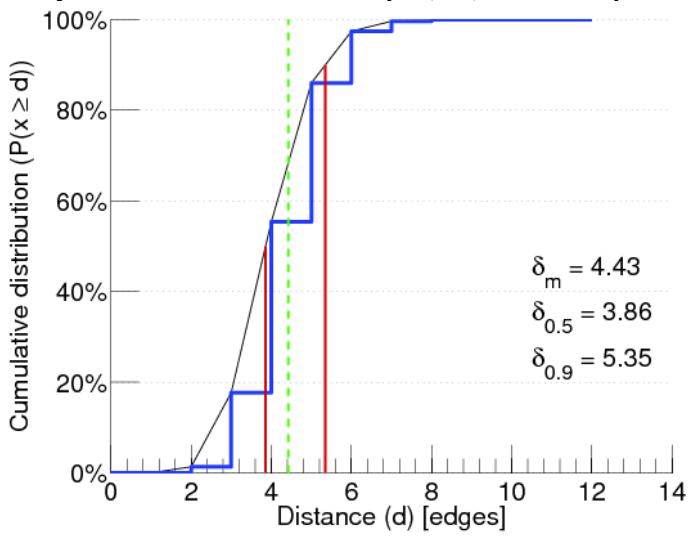




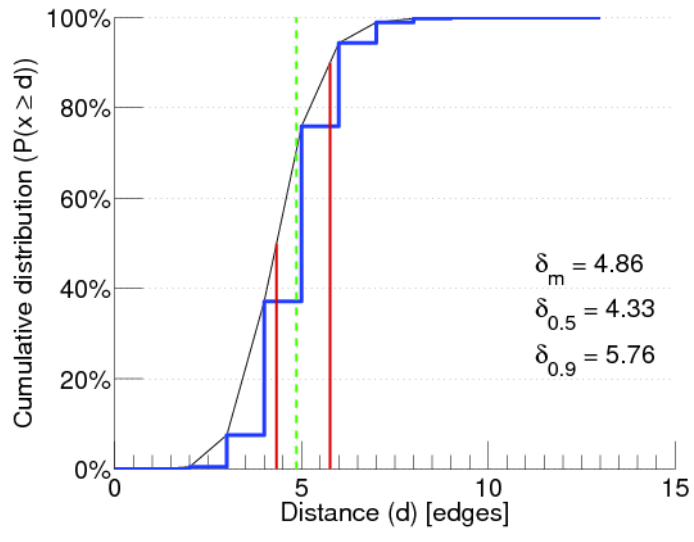

\section{Impact of datasets on location based social network}

Some datasets affecting LBSNs. The effects are identified with the measure of information gathered and its quality, its openness and perceivability, its feasible use by potential clients, and the level of wellbeing offered to the client by the application.

\subsection{Area information accumulation}

Area information accumulation signifies to the sort of area information gathered and put away alongside to its esteem. Foursquare collects and records client area information constantly and persistently, by speculating the client's present scope and longitude from the gadget being utilized. Client's registration into correct spots are tried in opposition to their normal present area and recorded expressly. Foursquare additionally expresses that it assembles included data from outsiders benefits, that interconnect with the demand, with individual data and exercises.

\subsection{Openness of area data}

Area data openness is worried about the amount of clients information are available and noticeable to others with the client, different clients and the outsiders of the administration. Clients pervious registration insights is conveyed to them as registration past where they can viewpoint their visited locales, date of the visit and any advices they have made [17]. They are additionally competent to right of passage and move their registration However, clients appear to have just a confined component of accessibility connected with what benefit provider can gather or adventure these information. Clients are just ready to square access to their registration and pictures by set their sentiment to 'private'. Concerning data revelation to outsiders and in addition Foursquare Programming interface's clients, the majority of the straightforwardly accessible clients' data is accessible by outsiders with private clients' data like registration in unspecified frame that isn't identified with discrete clients. Foursquare likewise determines that they will impart clients' close to home information to their colleagues and at whatever point is fundamental in a few circumstances, for example, execution of law.

\subsection{Abuse of area information}

Area data abuse speaks to how the application or outsiders can misuse the information and for which purposes. Foursquare gives itself finish rights over utilizing and working client data as demonstrated in their terms of utilization [18]. As of the abovementioned, obviously there are no commitments from the application provider about how the information may be utilized or else pooled by the application generally by different gatherings. In this manner, by supporting to the terms and conditions, clients proficiently are giving ceaselessly the information and unfit rights to utilize the information to the application.

\subsection{Security for area information}

Area information security wants to the level of information protection conveyed by the application for shielding the client's information in opposition to the danger of misfortune or else illicit access. Foursquare announces that the security of clients' data isn't distinct and any "Unapproved section or utilize, programming or equipment disappointment, and different variables, can trade off the wellbeing of client data whenever". Denied of any commitment to responsibility for information security, the application provider is announcing the conceivable high danger of information abuse by any challenger or even by the application provider themselves.

\section{Conclusion}

The objective of this article was to dissect a diagram of LBSNs datasets and information. In this review, we contemplate conduct of information in LBSNs, for example, area and security, abuse and openness. Furthermore, we talked about two kinds of online datasets to be specific Gowalla and Brightkite datasets and furthermore give examined in insight about the datasets. At that point the graphical investigation of the datasets likewise to be thought. At long last, the impact of datasets on area based informal community is profoundly examined. We trust that further research of client conduct in LBSNs will produce all the more intriguing examination issues and energizing arrangements around there.

\section{References}

[1] Valverde-Rebaza JC, Roche M, Poncelet P \& De Andrade Lopes A, "The role of location and social strength for friendship prediction in location-based social networks", Information Processing \& Management, Vol.54, No.4, (2018), pp.475-489.

[2] Bao J, Zheng Y, Wilkie D \& Mokbel MF, "A survey on recommendations in location-based social networks", ACM Transaction on Intelligent Systems and Technology, (2013), pp.1-30.

[3] Chen T, Kaafar MA \& Boreli R, "The Where and When of Finding New Friends: Analysis of a Location-based Social Discovery Network", ICWSM, (2013), pp.61-70.

[4] Yu Y \& Chen X, "A survey of point-of-interest recommendation in location-based social networks", Workshops at the Twenty-Ninth AAAI Conference on Artificial Intelligence, Vol.130, (2015), pp.53-60.

[5] Lian D, Zhu Y, Xie X \& Chen E, “Analyzing location predictability on location-based social networks", Pacific-Asia Conference on Knowledge Discovery and Data Mining, (2014), pp.102-113.

[6] Wang H, Terrovitis M \& Mamoulis N, "Location recommendation in location-based social networks using user check-in data", $21 s t$ ACM SIGSPATIAL International Conference on Advances in Geographic Information Systems, (2013), pp.374-383.

[7] Doan TN, Chua FCT \& Lim EP, "On neighborhood effects in location-based social networks", IEEE/WIC/ACM International Conference on Web Intelligence and Intelligent Agent Technology (WI-IAT), Vol.1, (2015), pp.477-484.

[8] Alrayes F \& Abdelmoty A, "Privacy concerns in location-based social networks", (2014), pp.1-10.

[9] Wang G, Schoenebeck SY, Zheng H \& Zhao BY, "Will Check-in for Badge; Understanding Bias and Misbehavior on Location-Based Social Networks. In ICWSM, (2016), pp.417-426.

[10] Persia F \& D'Auria D, "A Survey of Online Social Networks: Challenges and Opportunities", IEEE International Conference on Information Reuse and Integration (IRI), (2017), pp.614-620.

[11] Yousukkee S, "Survey of analysis of user behavior in online social network", IEEE International Conference on Management and Innovation Technology (MITicon), (2016), pp.MIT-128. 
[12] Zhang S, Zheng X \& Hu C, "A survey of semantic similarity and its application to social network analysis", IEEE International Conference on Big Data (Big Data), (2015), pp.2362-2367.

[13] Kumar H, Jain S \& Srivastava R, "Risk analysis of online social networks", IEEE International Conference on Computing, Communication and Automation (ICCCA), (2016), pp.846-851.

[14] Li N \& Chen G, "Analysis of a location-based social network", IEEE International Conference on Computational Science and Engineering, Vol.4, (2009), pp.263-270.

[15] Chang J \& Sun E, "Location 3: How users share and respond to location-based data on social networking sites", Fifth International AAAI Conference on Weblogs and Social Media, (2011), pp.74-80.

[16] Cho E, Myers SA \& Leskovec J, "Friendship and mobility: user movement in location-based social networks", 17th ACM SIGKDD international conference on Knowledge discovery and data mining, (2011), pp.1082-1090.

[17] Cranshaw J, Toch E, Hong J, Kittur A \& Sadeh N, "Bridging the gap between physical location and online social networks", Proceedings of the 12th ACM international conference on Ubiquitous computing, (2010) pp.119-128.

[18] Toch E, Cranshaw J, Drielsma PH, Tsai JY, Kelley PG, Springfield J, Cranor L, Hong J \& Sadeh N, "Empirical models of privacy in location sharing", 12th ACM international conference on Ubiquitous computing, (2010), pp.129-138. 